Check for updates

Kent

Cite this as: $B M J 2021 ; 374: n 2160$ http://dx.doi.org/10.1136/bmj.n2160 Published: 02 September 2021

\section{Covid-19: UK will offer third vaccine dose to severely immunosuppressed people}

\author{
Jacqui Wise
}

The UK's Joint Committee on Vaccination and Immunisation (JCVI) has recommended that people with severely weakened immune systems should have a third vaccine dose as part of their primary vaccination schedule against covid-19.

The third dose of either the Moderna or the Pfizer-BioNTech vaccine should be offered to people over age 12 who were severely immunosuppressed at the time of their first or second dose, including those with leukaemia, advanced HIV, or recent organ transplants. For 12-17 year olds the Pfizer-BioNTech vaccine is preferred.

The JCVI is still deciding on the benefits of booster doses for the rest of the population and is awaiting further evidence to inform this decision.

The committee has based its new advice on preliminary data from the Octave trial, which showed that almost everyone who was immunosuppressed did mount an immune response after two doses, as indicated by either antibodies or T cells-but in around $40 \%$ of people the levels of antibodies were low. It is not yet clear how much this may affect their protection against covid-19.

The JCVI has said that studies are ongoing to see how effective a third dose is in immunosuppressed people, but it is very unlikely to cause significant harms or disadvantages and may offer some benefit.

\section{Timing}

Wei Shen Lim, JCVI chair of covid-19 immunisation, said, "We want people with severely suppressed immune systems to have the best chance of gaining protection from covid-19 via vaccination. Therefore, we are advising they have a third vaccine dose on top of their initial two doses, as we hope this will reduce their risk of severe outcomes such as hospitalisation and death."

England's health and social care secretary, Sajid Javid, said that he accepted the JCVI's recommendations. "We know people with specific conditions that make them particularly vulnerable to covid-19 may have received less protection against the virus from two vaccine doses," he said. "I am determined to ensure we are doing all we can to protect people in this group, and a third dose will help deliver that."

Javid added, "This is not the start of the booster programme: we are continuing to plan for this to begin in September to ensure that the protection people have built from vaccines is maintained over time and ahead of the winter. We will prioritise those most at risk to covid-19, including those who are eligible for a third primary vaccine, for boosters based on the final advice of the JCVI."

The decision on the timing of the third primary dose should be taken by the specialist involved in the care of the patient, the JCVI said. In general, the timing should usually be at least eight weeks after the second dose, but there should be some flexibility. For example, it is preferable to give a vaccine dose before someone undergoes chemotherapy rather than during the treatment.

People with less serious immunosuppression are not included in the latest advice but are likely to become eligible for another dose as part of any potential booster programme. If the JCVI gives the go-ahead for a booster programme severely immunocompromised people will also be offered a booster dose at a suitable interval after their third dose. 